\title{
PROCESSO DE TRABALHO DOS ENFERMEIROS DE UM HOSPITAL DA ESPANHA: ÊNFASE NAS TECNOLOGIAS DE CUIDADO*
}

Maira Buss Thofehrn', Maria José Lopes Montesinos², Isabel Cristina Arrieira², Veridiana Corrêa Àvila4, Tânia Cristina Schäfer Vasques ${ }^{5}$, Izamir Duarte de Farias $^{6}$

\begin{abstract}
RESUMO: O estudo de natureza qualitativa, descritiva e exploratória objetivou conhecer o processo de trabalho dos enfermeiros de um Hospital Universitário de Múrcia - Espanha. Participaram oito enfermeiros, os quais desenvolviam atividades assistenciais e de gestão. As informações foram obtidas no período de novembro de 2011 a janeiro de 2012 por meio de auto-observação e descrição, pelos próprios enfermeiros, em relação ao entendimento sobre seu processo de trabalho. Os textos produzidos foram enviados por e-mail para o grupo de pesquisadores e os dados analisados a partir da análise temática, emergindo os temas: a importância da passagem de plantão enquanto tecnologia de cuidado e ações dos enfermeiros com vistas ao desenvolvimento de uma tecnologia do cuidado. Evidenciou-se que o enfermeiro atua como pilar no processo de trabalho, assumindo responsabilidades em busca de tecnologias para executar o cuidado. DESCRITORES: Trabalho; Hospitais universitários; Enfermagem; Tecnologia.

\section{PROCESO DE TRABAJO DE LOS ENFERMEROS DE UN HOSPITAL DE ESPAÑA: ENFASIS EN LAS TECNOLOGÍAS DE CUIDADO}

RESUMEN: Este estudio de naturaleza cualitativa, descriptiva y exploratoria tuvo el objetivo de conocer el proceso de trabajo de los enfermeros de un Hospital Universitario de Murcia - España. Participaron ocho enfermeros, los cuales desarrollaban actividades asistenciales y de gestión. Las informaciones fueron obtenidas en el periodo de noviembre de 2011 a enero de 2012 por medio de auto observación y descripción, por los propios enfermeros, acerca del entendimiento sobre su proceso de trabajo. Los textos producidos fueron enviados por e-mail para el grupo de investigadores y los datos analizados por medio del análisis temático, resultando los temas: la importancia de la pasaje de guardia mientras tecnología de cuidado y acciones de los enfermeros para el desarrollo de una tecnología del cuidado. Se evidenció que el enfermero actúa como amparo en el proceso de trabajo, asumiendo responsabilidades en búsqueda de tecnologías para ejecutar el cuidado.

DESCRIPTORES: Trabajo; Hospitales universitarios; Enfermería; Tecnología.

\section{NURSES' WORK PROCESS IN A HOSPITAL IN SPAIN: EMPHASIS ON THE TECHNOLOGIES OF CARE*}

\begin{abstract}
This descriptive and exploratory study with a qualitative character aimed to investigate the work process of the nurses in a University Hospital in Murcia, Spain. Eight nurses participated, who undertook care and management activities. The information was obtained in the period November 2011 - January 2012 through self-observation and description, by the nurses themselves, in relation to their understanding of their work process. The texts produced were sent to a group of researchers by email and the data were analyzed using thematic analysis, with the following themes emerging: the importance of end of shift handover as a technology of care, and actions of the nurses with views to the development of a care technology. It was evidenced that the nurse functions as a cornerstone in the work process, taking on responsibilities in the search for technologies for carrying out the work. DESCRIPTORS: Work; University hospitals; Nursing; Technology.
\end{abstract}

\footnotetext{
*Artigo original desenvolvido a partir do estágio pós-doutoral realizado em Múrcia - Espanha, com auxílio financeiro da CAPES.

${ }^{1}$ Enfermeira. Doutora em Enfermagem. Professora da Universidade Federal de Pelotas. Bolsista CAPES de estágio Pós-doutoral na Faculdade de Múrcia.

${ }^{2}$ Enfermeira. Doutora em Enfermagem. Professora da Universidade de Múrcia.

${ }^{3}$ Enfermeira. Mestre em Ciência da Saúde. Professora na Faculdade de Enfermagem da Universidade Católica de Pelotas. Doutoranda em Enfermagem. Pelotas-RS-Brasil

${ }^{4}$ Enfermeira. Associação de Caridade Santa Casa de Rio Grande.Rio Grande-RS-Brasil

${ }^{5}$ Enfermeira. Mestre em Enfermagem. Universidade Federal de Pelotas. Pelotas-RS-Brasil

${ }^{6}$ Técnico Superior em Artes. Mestre em Ciências da Saúde. Tutor do Curso de Especialização em Mídias na Educação EaD. Universidade Federal de Pelotas. Pelotas-RS-Brasil
} 


\section{INTRODUÇÃO}

A enfermagem enquanto profissão da saúde é reconhecida desde a segunda metade do século XIX, a partir de Florence Nightingale que normatizou um campo de atividades de cuidado à saúde que até então eram desenvolvidas por pessoas ou grupos com diferentes qualificações e cenário. O cuidado agrega especialidade no conjunto da divisão do trabalho social, sendo reconhecido como um campo de atividades especializadas necessárias para a sociedade e que, para o seu exercício, requer formação especial e produção de conhecimentos que fundamentem a ação destes profissionais ${ }^{(1)}$.

Estes conhecimentos lhes dão competências para cuidar das pessoas, em todo o seu processo de viver, educar e pesquisar que inclui o ato intrínseco ao processo de cuidar, envolvendo a educação permanente no trabalho, a formação de novos profissionais e a produção de conhecimentos que subsidiem o processo de cuidar. Salienta-se ainda a dimensão administrativa de coordenação do trabalho coletivo da enfermagem, de gerenciamento do espaço assistencial, de participação na gestão da atenção à saúde e no gerenciamento institucional ${ }^{(2)}$. Portanto, a qualidade nas ações de enfermagem reflete na qualidade da assistência em saúde.

Nesse sentido, a perspectiva da tecnologia do cuidado como atividade profissional representa reflexão importante para a enfermagem, contemplando parte significativa de suas ações, com isso possibilita a reflexão do processo de trabalho, de forma ampla e também valoriza as competências profissionais necessárias para a prestação do cuidado ${ }^{(3)}$.

Desta forma a produção da tecnologia é essencial para as inovações na área da saúde e enfermagem, pois auxilia a organização do serviço, as formas de cuidar, fundamenta e inova a prática do exercício profissional. A produção e utilização da tecnologia ocasionam uma postura reflexiva e pautada em princípios éticos para a tomada de decisão profissional. Além disso, os resultados da sua utilização permeiam o contexto da prática da enfermagem ${ }^{(3)}$.

Assim, o processo de trabalho na saúde, mesmo sendo um serviço que desencadeia atividade humana, também opera uma transformação, subordinada a um determinado fim, no objeto sobre o qual atua por meio do instrumental ${ }^{(4)}$. O objeto de trabalho não se transforma em outro, mas na promoção de saúde às pessoas que procuram as organizações hospitalares.

Nesse foco, a enfermagem, por meio da visão sistêmica do enfermeiro, tem condições de mobilizar a equipe efetivando o processo de cuidar inserindo a tecnologia. Assim, seus resultados e impactos dependerão do conhecimento e reflexão do profissional, os quais norteiam a forma como ela será utilizada. Portanto, estabelece a ação do profissional em executar o cuidado $^{(3)}$.

O desenvolvimento de tecnologia do cuidado envolve três formas de tecnologias presentes no processo produtivo de trabalho. Adaptados a este estudo, correspondem as tecnologias duras, que são os materiais, equipamentos, máquinas e demais instrumentais utilizados durante a realização do cuidado. Também as tecnologias leve-duras, entendidas como todo o conhecimento e metodologias assistenciais na execução do cuidado. E, por fim as tecnologias leves ou relacionais que se fazem necessárias no entorno da ação de cuidar ${ }^{(5)}$.

Desta forma, a enfermagem precisa tomar posse e reconhecer que seu trabalho é imprescindível no cuidado aos pacientes, tornando viável a implementação das tecnologias do cuidado, com a busca de inovações, com uma visão crítica sobre o fazer podendo tornar possível uma transformação da realidade cotidiana. Assim, possibilita maior visibilidade desses profissionais, que por diversas vezes não são reconhecidos nos seus afazeres diários, melhorando sua qualidade de vida e satisfação pessoal ${ }^{(6)}$.

Assim, este estudo teve como objetivo conhecer o processo de trabalho dos enfermeiros de um Hospital Universitário de Múrcia - Espanha quanto às tecnologias de cuidado.

\section{MÉTODO}

Este estudo caracteriza-se por apresentar uma abordagem qualitativa, descritiva e exploratória, o qual atendeu às recomendações éticas para estudos envolvendo seres humanos; foi realizado após o parecer favorável do comitê de ética sob parecer n. 191/2012. A pesquisa foi desenvolvida por meio de auto-observação e descrição da rotina de trabalho dos enfermeiros que atuam em diferentes áreas do Hospital Geral Universitário Rainha Sofia na cidade de Múrcia - Espanha. O referido hospital comporta 320 leitos distribuídos em Unidade de Emergência, Centro Cirúrgico, Unidade de Terapia Intensiva, Unidade de Internação tais como cardiologia, pneumologia, entre outros.

Foram participantes do estudo oito enfermeiros das Unidades de Internação, Unidade de Terapia Intensiva, Unidade de Emergência e enfermeiros em cargos ges- 
tores do serviço de enfermagem. A equipe é composta pelo enfermeiro gerente da unidade e auxiliares de enfermagem, sendo que os auxiliares não podem atuar sem a presença do enfermeiro e a competência dos mesmos está relacionada a procedimentos de menor complexidade, tais como higiene e conforto do paciente.

A coleta dos dados ocorreu nos meses de novembro de 2011 à janeiro de 2012. Foi realizado um encontro com 12 enfermeiros da instituição hospitalar em questão, em que foram apresentados os objetivos do estudo e os mesmos instigados a refletirem sobre o seu processo de trabalho. Após esse processo, oito enfermeiros aceitaram o desafio de descreverem suas atividades de um dia de trabalho, sendo esta descrição encaminhada ao grupo de pesquisadores via email. Os participantes foram identificados pela letra "E" seguida pelo número ordinal correspondente a ordem de realização do processo da pesquisa.

Os dados foram tratados por intermédio da Análise Temática ${ }^{(7)}$ e da qual emergiu os seguintes temas: importância da passagem de plantão enquanto uma tecnologia de cuidado e ações dos enfermeiros com vistas ao desenvolvimento de uma tecnologia do cuidado.

\section{RESULTADOS}

Ao analisar o perfil dos oito enfermeiros participantes do estudo, observou-se que a idade variou de 32 anos a 53 anos; em relação ao tempo de formação alternou entre 7 e 27 anos, e de trabalho na instituição entre 4 e 25 anos. Além disso, cinco desenvolviam atividades que se caracterizam como assistenciais e três em cargo de gerenciamento. Ainda, salienta-se que apenas um dos enfermeiros, entre os oito participantes, possuia especialização e mestrado.

\section{A importância da passagem de plantão enquanto uma tecnologia do cuidado}

Considerando que o cuidado de enfermagem hospitalar caracteriza-se por ter caráter ininterrupto, o encontro do enfermeiro que está saindo de seu turno de trabalho com o que está assumindo o referido trabalho se torna a garantia desta continuidade. Para tanto, pode-se perceber a importância da comunicação na passagem de plantão, tanto verbal quanto escrita, para que haja continuidade do cuidado ao paciente com coerência, de maneira a dar efetivamente continuidade aos procedimentos da equipe anterior, conforme identificado nas falas a seguir:
Oenfermeiro do turno anterior nos indica o diagnóstico do paciente, situação das últimas horas, se tem algum exame pendente para realizar, situação da família. (E2)

Realiza-se a passagem de plantão da forma conjunta com o pessoal auxiliar de enfermagem. Tem uma duração de 10-15 minutos, conhecemos o estado clínico do paciente, comunicamos os problemas detectados ou os resolvidos e também nos permite organizar junto ao auxiliar as tarefas a desempenhar de forma conjunta durante o turno. (E3)

Percebe-se comprometimento dos enfermeiros para com a passagem de plantão, demonstrando valorização de seu fazer, como exemplificado abaixo:

Realizo a descrição das atividades da unidade do hospital, todos os turnos iniciam igual, dividindo informação essencial de cuidados entre a enfermeira que sai e a enfermeira que chega, é uma intervenção importantissima e marca decisivamente a jornada de trabalho. (E5)

São transmitidos dados objetivos, que quantificamos em nossos gráficos, assim como de impressões pessoais que nossos companheiros nos transmitem, que nos orientam em nosso trabalho posterior. (E8)

Em contrapartida, quando essa comunicação não é efetiva, muitas vezes, o profissional do turno seguinte não visualiza o trabalho feito com o paciente naquele turno, podendo provocar a frustração do profissional. Este se sente desanimado pela não continuidade do cuidado por parte de seus colegas, como pode ser visto na fala, a seguir:

Se eu considero que os cuidados no turno não foram adequados, gera em mim um grande sentimento de pesar pelo paciente e desapontamento com relação a minha colega e me questiono sobre o significado de "trabalho em equipe" e "atenção continuada". (E4)

\section{Ações dos enfermeiros com vistas ao desenvolvi- mento de uma tecnologia do cuidado}

Comumente tem-se observado que os enfermeiros assumem diversas atividades, fazendo com que estes tenham uma visão de todo andamento do serviço de enfermagem, envolvendo-se com o cuidado, enquanto uma tarefa profissional, conforme observado nos depoimentos a seguir: 
Preparar a medicação prescrita, administrar no turno, coletar exames de sangue, revisar os dispositivos terapêuticos invasivos e não invasivas, promover a deambulação, higiene e revisão da integridade cutânea do paciente; registramos todas as atividades e intercorrências de enfermagem no computador. (E3)

Intervenções sobre cuidados de feridas cirúrgicas, manejo da dor, cuidados de drenagem. (E6)

Revisamos os diferentes box de assistência, repomos a medicação e material, também o funcionamento correto dos aparelhos de atenção de emergências, assim como, a medicação e material do carro de parada. Então, passamos a realizar o trabalho de cateterização de acessos venosos periféricos. (E7)

Ainda verificou-se, por meio das falas, que a rotina de trabalho do enfermeiro versa desde a atividade gerencial, a assistencial e educacional.

\section{Atenção aos alunos que passam pela unidade. (E1)}

Eu gostaria de passar menos tempo em atividades burocráticas ou em intermináveis reuniões (muitas delas improdutivas) e estar mais pelas unidades, perto da enfermagem assistencial, acho que isso me permitiria conhecer melhor a realidade e que linhas estratégicas deveria desenvolver para melhorar a qualidade assistencial que se presta os usuários. (E2)

Anoto na pasta o motivo da consulta, os dados que mensurei, confiro se o paciente tem a pulseira de identificação, entrego uma autorização para que uma familiar possa entrar acompanhando o paciente. (E8)

Outro aspecto também importante no fazer diário dos enfermeiros consiste em preparar ambientes e condições favoráveis para a execução e manutenção de procedimentos, em que as tecnologias leve-duras podem ser evidenciadas. Com o intuito de proporcionar uma maior qualidade na assistência, por meio do conhecimento técnico-científico adquirido pelos enfermeiros, proporcionando bem estar ao paciente. Tais colocações se evidenciam nas falas a seguir:

Atendemos às campainhas, o telefone e planejamos junto a outros profissionais a preparação dos quartos para internações hospitalares. (E1)
Participar na visita médica, avaliação contínua do bem-estar do paciente, do conforto em geral. (E4)

\section{DISCUSSÃO}

\section{A importância da passagem de plantão enquanto uma tecnologia do cuidado}

É fato que a passagem de plantão representa o momento de reatualização da informação e continuidade dos cuidados, tendo também a função de manter unidos os enfermeiros e a equipe de enfermagem. De certo modo, revela a expressão do cooperativismo profissional, assumindo, nesse momento, uma representação suprema e compartilhada pelos pares ${ }^{(8)}$.

Dessa maneira, a passagem de plantão se torna um procedimento primordial no trabalho de um enfermeiro, independente do local, país, cultura ou setor em que exerce suas funções. Ainda é compreendido como o momento de conhecimento do paciente, seu diagnóstico, intercorrências clínicas e de enfermagem ${ }^{(9)}$. Assim, para este estudo fica evidenciado como o primeiro passo no desenvolvimento de uma tecnologia para o cuidado.

Para tanto a comunicação torna-se uma ferramenta importante, sendo valorizada e essencial em todo esse processo, envolvendo a troca de informações, o relacionamento e a compreensão do saber, que são transmitidas na passagem de plantão ${ }^{(9)}$. Dessa forma, a mudança de turno é uma prática na qual se utiliza da comunicação como instrumento básico de enfermagem, com vistas a transmitir informações objetivas, claras e concisas sobre os acontecimentos ocorridos durante um período de trabalho, envolvendo o cuidado direto e indireto ao paciente bem como assuntos de interesse institucional ${ }^{(10)}$.

Fica evidente que esse tempo, em que os profissionais se encontram para socializar as informações referentes ao plantão, é crucial para que o turno seguinte possa ser bem sucedido, pois o que ocorre durante cada momento de trabalho da equipe de enfermagem refere-se à evolução do paciente, o que em muitos casos pode ser fundamental para a vida do paciente. Quando ocorre inadequação na prestação do cuidado pelo turno anterior também é na passagem de plantão que se torna possível equacionar tais divergências, até para que se consiga a formação de uma equipe de enfermagem com vínculos saudáveis e assim garantir a continuidade do cuidado.

Então, nessa vertente, o profissional enfermeiro torna-se o elo nos relacionamentos, garantindo que as 
relações humanas sejam harmoniosas e produtivas, para que a finalidade ou objetivo do trabalho seja o cuidado com qualidade para todos os envolvidos ${ }^{(11)}$. Em suma, a passagem de plantão deve ser vista como um momento que transcende uma simples troca de informação, e que possa servir como momento de reflexão e avaliação do trabalho interdisciplinar, objetivando o crescimento mútuo e o melhor desempenho do trabalho em saúde ${ }^{(10)}$.

\section{Ações dos enfermeiros com vistas ao desenvolvi- mento de uma tecnologia do cuidado}

Ao analisar as atividades assumidas pelos enfermeiros, cabe a esse profissional desenvolver habilidades no seu processo de cuidar, envolvendo atitudes e comportamentos que se baseiam em conhecimento científico, técnico, pessoal, cultural, psicológico e espiritual, no intuito de se obter um olhar abrangente no seu campo de atuação ${ }^{(6)}$.

Dessa forma, o cuidado em enfermagem é considerado a essência dessa profissão, sendo esse cuidar dividido em duas vertentes: a objetiva, englobando técnicas e procedimentos e a subjetiva, guiada pela intuição e percepção dos profissionais da enfermagem ${ }^{(6)}$. Portanto, as tarefas dos enfermeiros versam desde o seu fazer prático, como o teórico e relacional. Assim, no seu fazer diário, ele passa a utilizar as tecnologias mesmo sem perceber, em que podemos citar: as tecnologias duras, com o uso de equipamentos, aparelhos; ou com a utilização de procedimentos, com conhecimento específico, podendo ser classificadas de tecnologias leve-duras, como é o caso da utilização de metodologia de assistência em enfermagem e também o uso de tecnologias leves, com foco nas relações interpessoais, em que o enfermeiro tem como objeto de seu trabalho, sua equipe e seus pacientes ${ }^{(6)}$.

Nesse contexto, o cuidado, enquanto tarefa profissional, corresponde à ação da enfermagem enquanto tecnologia para o alcance de um fim. Assim, tal tarefa pode ser reconhecida como tecnologia de cuidado, pois a ação de enfermagem somente pode se efetivar a partir de embasamento teórico e de habilidade técnica ${ }^{(12)}$.

Dessa forma, o reconhecimento das atividades desenvolvidas pelos enfermeiros no cotidiano das instituições é fundamental, visto que possibilita visualizar as lacunas existentes no seu trabalho e, consequentemente, auxilia na delimitação de suas funções. Além disso, podem trazer elementos que contribuam para o desempenho de ações mais criativas, inovado- ras, participativas e mais próximas do paciente e do trabalhador favorecendo o conhecimento das reais necessidades dos envolvidos neste processo de traba1 ho ${ }^{(13)}$. No entanto, possivelmente pela diversidade de afazeres que compete a enfermagem, muitas vezes as tecnologias ditas leves são pouco trabalhadas na prática pelos enfermeiros no seu cuidado diário(11).

Os enfermeiros também dedicam seu tempo às atividades educativas referentes ao sistema de informação que são relevantes para a sistematização da assistência e funcionamento do serviço ${ }^{(13)}$. Para tanto, esses profissionais investem na educação dos estudantes, quanto a seu processo critico-reflexivo, propondo formas de educar inovadoras e criativas, preocupando-se com o processo de educar e os valores que estão sendo construídos por eles ${ }^{(14)}$.

Ainda na função de educadores, esses trabalhadores também orientam a população para que se tornem agentes de promoção e proteção de sua saúde e da comunidade em que está inserida, em uma relação horizontal, de igualdade. Portanto há um processo de conscientização dos sujeitos para obterem maior controle sobre suas vidas ${ }^{(15)}$.

É pertinente a reflexão contínua dos enfermeiros sobre as ações de cuidado para que este trabalhador passe assumir seu núcleo de atuação e exercer a autonomia, recebendo reconhecimento e valorização do seu trabalho ${ }^{(16)}$. Além desse contribuir no fortalecimento e consolidação da enfermagem enquanto ciência e tecnologia ${ }^{(17)}$.

Nesse contexto, perceber-se que a tecnologia de cuidado na enfermagem pode tornar-se instrumento para fortalecer e qualificar o mesmo. Dessa maneira, ao se falar em tecnologia se remete a procedimentos de conhecimento e instrumentos interligados de forma sistematizada do saber-fazer ${ }^{(6)}$. Assim, o cuidado aos pacientes é algo que se torna mais efetivo quando incorpora os processos e as expressões tecnológicas do conhecimento, com a finalidade de um trabalho vivo em ato, sistematizado e organizado ${ }^{(6)}$.

\section{CONSIDERAÇÕES FINAIS}

O estudo mostra que os enfermeiros atuam de forma ampliada, iniciando suas jornadas a partir do recebimento do plantão do turno anterior, sendo este importante ato para a contextualização da realidade e planejamento das ações. Desta forma podemos afirmar que a tecnologia do cuidado na Espanha tem seu ponto de partida na passagem de plantão, a qual ocorre de 
modo detalhado e comprometido com os pacientes e com a equipe de enfermagem.

No que diz respeito às atividades desempenhadas pelos enfermeiros, observou-se que estão relacionadas à assistência direta aos pacientes, cuidados com a ambiência e atividades com os alunos de graduação em enfermagem. Destacou-se também a importância do trabalho em equipe, sendo em alguns casos relatados pelos enfermeiros como primordial para o bom funcionamento da unidade e consequentemente para a qualidade da assistência.

Ainda ficou evidente que pelo enfermeiro passam todas as decisões pertinentes ao cuidado dos pacientes e atenção à família, atuando como o pilar de referência para a equipe estando presente e atuante em cada passo do processo de trabalho. Os enfermeiros também demonstraram interesse pela busca contínua de conhecimento, possibilitando sucessivo desenvolvimento da profissão com a visão de integralidade da atenção aos pacientes e as famílias.

O estudo proporcionou a compreensão do processo de trabalho de enfermeiros que atuam em hospital geral na Espanha, possibilitando-nos ampliar nossa visão em relação aos espaços ocupados por estes profissionais no cenário internacional.

\section{REFERÊNCIAS}

1. Pires D. A enfermagem enquanto disciplina, profissão e trabalho. Rev. bras. enferm. 2009;62(5):739-44.

2. Pires D, Matos E. Teorias administrativas e organizações do trabalho: de Taylor aos dias atuais, influências no setor saúde e na enfermagem. Texto Contexto Enferm. 2006;15(3):508-14.

3. Crozeta K, Stocco JGD, Lambronici LM, Meier MJ. Interface entre a ética e um conceito de tecnologia em enfermagem. Acta Paul. Enferm. 2010;23(2):239-43.

4. O capital: Crítica da economia política. Trad. Reinaldo Sant'anna. Rio de Janeiro: Civilização Brasileira; 2011.

5. FrancoTB, Merhy EE. Cartografias do Trabalho e Cuidado em Saúde. Rev. Tempus Actas Saúde Colet. 2012;6(2):151-63.

6. Rocha PK, Prado MLP, Wal ML, Carraro TE. Cuidado e tecnologia: aproximações através do Modelo de Cuidado. Rev .bras. enferm. 2008;61(1):113-6.

7. Minayo MCS. O Desafio do Conhecimento: Pesquisa Qualitativa em Saúde. 12a ed. São Paulo: Editora
Hucitec/Rio de Janeiro: ABRASCO; 2010.

8. Penaforte MHO. Martins MMFP. A visibilidade do autocuidado relativo à higiene na passagem de plantão dos enfermeiros. Rev. Latino-Am. Enfermagem. 2011;19(1):1-9.

9. Rodriguez L, Oliveira EO, de França CS, de Andrade TRS, Campos JSPAC, Silva MP, Costacurta FJ. Mapeamento da passagem de plantão sob a ótica dos profissionais de enfermagem. Enfermería Global. 2013;31:219-31.

10. Silva EE, Campos LF. Passagem de plantão: revisão da literatura. Cogitare enferm. 2007;12(4):502-7.

11. Pereira CDFD, Pinto DPSR, Tourinho FSV, Santos VEP. Tecnologias em enfermagem e o impacto na prática assistencial. Revista Brasileira de Inovações Tecnológicas em Saúde. [Internet] 2012;2(4) [acesso em 15 set 2013]. Disponível: http://ufrn.emnuvens.com.br/ reb/article/view/3331

12. Thofehrn MB, Leopardi MT. Teoria dos vínculos profissionais: Um novo modo de gestão em enfermagem. Texto Contexto Enfermagem. 2006;15(3):409-17.

13. Costa RA, Shimizu HE. Atividades desenvolvidas pelos enfermeiros nas unidades de internação de um hospital-escola. Rev. Latino-Am. Enfermagem. 2005;13(5):654-62.

14. Jesus MCP, Santos SMR, Merighi MAB, Oliveira DM, Figueiredo MAG, Braga VA. Vivência do estudante de enfermagem em atividades de educação em saúde. Cienc. cuid. saude. 2012;11(3):436-44.

15. Monteiro MAA, Oliveira SHS, Pinheiro AKB, Ximenes LB, Barroso MGT. Promoção da saúde de puérperas: conhecimento e práticas de enfermeiras. Rev Rene. 2012;13(2):280-90.

16. Persegona KR, Rocha DLB, Lenardt MH, Zagonel IPS. O conhecimento político na atuação do enfermeiro. Esc. Anna Nery. 2009;13(3):645-50.

17. Ministério da Saúde(BR). Conselho Nacional de Saúde. Regulamento do Sistema Único de Saúde.Portaria 2048/2009. Brasília (DF): MS; 2009. 